\title{
CAPACIDAD DE DISFRUTE Y PERCEPCIÓN DEL APOYO COMUNITARIO EN ADOLESCENTES ESPECTADORES DE EPISODIOS DE VIOLENCIA ENTRE PARES (BULLYING)
}

\author{
ABILITY TO ENJOY THE COMMUNITY SUPPORT AND PERCEPTION \\ OF VIOLENCE AMONG VIEWERS IN PAIRS (BULLYING) \\ Alberto Quintana P.; William Montgomery U.; Carmela Malaver S.; Gabriela Ruiz S. \\ Universidad Nacional Mayor de San Marcos, Perú \\ (Recibido el 25/02/2010, ACEPTAdo el 22/06/2010)
}

\begin{abstract}
RESUMEN
En una muestra de adolescentes espectadores de episodios de violencia entre pares se estudian las relaciones entre la capacidad de disfrute del individuo y su percepción del apoyo comunitario en la escuela, a fin de verificar si dichas variables se correlacionan positivamente entre sí, y de que existen diferencias significativas entre los tipos de espectadores de episodios de violencia entre pares en relación a las mismas. Para ello se utilizaron como indicadores operacionales de estos procesos: la escala de gaudibilidad de Padrós, la escala de apoyo comunitario percibido adaptadas al ambiente escolar, y la escala de tipo de espectador de violencia entre pares validada en un estudio anterior. Se encontró correlaciones positivas significativas entre la capacidad de disfrute y la percepción subjetiva de apoyo comunitario en la escuela que tienen los adolescentes, así como con los distintos componentes de esta última. Igualmente que existen diferencias significativas, en la capacidad de disfrute del individuo y la percepción del apoyo comunitario en la escuela, entre el tipo de espectador prosocial y los tipos de espectador amoral e indiferente culpabilizado, no encontrándose diferencias significativas en cuanto a estos procesos entre estos últimos.
\end{abstract}

Palabras clave: Escuela; adolescentes; violencia entre pares (bullying); espectadores; capacidad de disfrute; percepción de apoyo comunitario.

\begin{abstract}
In a sample of adolescent viewers of violence between couples studied the relations between the ability to enjoy the individual and their perception of community support in school, in order to verify whether these variables are positively correlated with each other, and that there Significant differences between the types of viewers of violence between couples in relation to them. This was used as operational indicators of these processes: Scale Padrós Gaudiability, the Scale of Perceived Community Support tailored to the school environment, and Type Scale Peer Violence Viewer validated in a previous study. We found significant positive
\end{abstract}

1 Docente principal de la Facultad de Psicología de la UNMSM.

E-mail: albertolqp@yahoo.es 
correlations between the capacity of enjoyment and perceptions of community support in school among adolescents, as well as the various components of the latter. Equally significant differences in the ability to enjoy the individual and the perception of community support in school, between the viewer and the types of prosocial amoral and indifferent spectator blame, but there were no significant differences between these processes past.

Keywords: School; teenagers; peer violence (bullying); spectators; capacity of enjoyment; perception of community support.

\section{INTRODUCCIÓN}

La ideología de los estados mórbidos, la enfermedad, ha dominado el estudio de la salud en la psicología (Blanco y Díaz, 2006). Sin embargo, la Organización Mundial de la Salud (OMS) ha acentuado el interés en las condiciones subjetivas que permitirían conseguir mejores niveles de bienestar psicológico y social. Dentro de tales condiciones son fundamentales el optimismo, la creatividad, la capacidad de disfrute, el humor, la empatía y diversas capacidades de afrontamiento, estudiadas por la psicología positiva (Seligman y Csikszentmihalyi, 2000).

En la investigación acerca de los modos de afrontamiento y conducta resiliente en adolescentes espectadores de violencia entre pares (Quintana, Montgomery y Malaver, 2009) se encontró que el espectador prosocial, aquel que enfrenta activamente la situación confrontando al victimario y/o solicitando ayuda de otros compañeros o docentes, es el que más frecuentemente recurre a estrategias tales como afrontamiento activo, de planificación y búsqueda de apoyo social por razones instrumentales y emocionales, entre otros.

El espectador prosocial es aquel que se muestra significativamente más resiliente; es decir, que entre otras cosas se caracteriza por un adecuado control de emociones en situaciones difíciles, por el optimismo y una mayor tendencia a manifestar persistencia ante el fracaso y sentimientos de esperanza sumados a la capacidad de enfrentar activamente los problemas cotidianos.

Interpretando estos resultados en términos de la psicología positiva, se podría decir que el espectador prosocial es el tipo de espectador que probablemente percibe un mayor apoyo comunitario (Gracia y Herrero, 2004), y a la vez presenta una mayor capacidad de disfrute o "gaudibilidad" (Padrós, 2002; Padrós y Fernández, 2002).

Por ello, el presente estudio pretende ser una contribución para especificar, en los adolescentes espectadores de episodios de violencia entre pares, el tipo de comportamiento prosocial, la capacidad de disfrute y la percepción subjetiva de apoyo comunitario que permitirían sustentar una alternativa de intervención preventiva de la problemática de la violencia entre pares, favoreciendo además la promoción de una salud integral. Todo ello en el contexto de la declaración de la UNESCO sobre el desarrollo de una cultura de paz y no violencia. Convicciones que también se han visto reflejadas en las directivas formuladas por la Asociación de psicología americana en el decenio de la Década de la conducta (2000-2010), y así mismo en el Plan nacional de salud publicado por el Ministerio de Salud del Perú desde el año 2005. 
Es conocido que el asunto de los fundamentos psicológicos de "la buena vida", del disfrute y del bienestar subjetivo se remonta, hasta la época antigua. Se habla de controversias entre las escuelas estoica, hedonista y aristotélica respecto a la felicidad, no obstante, coinciden con matices en lo principal. En particular, la "felicidad suma" era para Aristóteles el objetivo del buen vivir, y Arístipo, desde la perspectiva hedonista, consideraba que dicha meta se cumplía solo si se experimentaba la mayor cantidad de placer posible (Padrós, 2002). Por otro lado, Confucio y sus discípulos identificaban una feliz calidad de vida con la vida de un ciudadano en una sociedad ordenada, con responsabilidades claras.

Ya establecida la psicología como disciplina, su orientación terapéutica se vinculó principalmente al estudio de los estados mórbidos, obviando las emociones positivas. Recientemente, se retoma el interés por aquellas, postulando conceptos como la autoactualización, el funcionamiento pleno y la madurez, vinculados al individuo como agente de cambio (Díaz Aguado, 2006). En esta línea, la psicología positiva (Seligman y Csikszentmihalyi, 2000) refleja la actual filosofía desplegada por la OMS, acentuando el interés en las condiciones subjetivas que permitirían conseguir mejores niveles de bienestar en las dimensiones psicológica y social. Dentro de ellas, juegan un papel fundamental el optimismo, la creatividad, la capacidad de disfrute, el humor, la empatía y diversas capacidades de afrontamiento. Todas ellas vinculadas a la relación entre las características del ambiente (dentro del cual cuenta el número y calidad de las relaciones sociales), y el nivel de satisfacción experimentado por las personas, siendo además indicadores subjetivos de la calidad de vida (Vázquez, Hervás y Ho, 2006; Salotti, 2006).

El interés de este estudio está centrado, en primer lugar, en la capacidad de disfrute o "gaudibilidad" (Padrós, 2002) y la percepción subjetiva de apoyo comunitario (Gracia y Herrero, 2006), en el entendido de que constituyen variables centrales en el concepto de bienestar "alejado de esa ideología de la enfermedad que ha dominado el estudio de la salud en la psicología, de una salud (...) protagonizada por un sujeto suspendido en el vacío" (Blanco y Díaz, 2006, p. 11). Siguiendo ese razonamiento se intenta explorar si la capacidad de disfrute y la percepción subjetiva de apoyo comunitario están relacionadas debido a sus características de variables topográficas y funcionales relativas al individuo como agente de su propia conducta (y por ende de su propia salud), y a la vez, implicado en los problemas que aquejan a la sociedad a la cual pertenecen.

En este contexto, considerando la incidencia frecuente de episodios de violencia entre pares (acoso escolar), y el papel que juegan los espectadores de tales episodios por su carácter de fuente de reforzamiento social, y en la búsqueda de conceptualizaciones que permitan desarrollar pautas efectivas de prevención y programas de tratamiento de la violencia, consideramos importante relacionar también las variables de capacidad de disfrute y percepción de apoyo comunitario con la tendencia prosocial o antisocial de tales espectadores. Cabe notar que en estudios previos (Avilés, 2006; Quintana; Montgomery y Malaver, 2009) se han encontrado diferencias significativas en la capacidad de afrontamiento entre los tipos de adolescentes involucrados como espectadores en episodios de acoso; por ello es interesante verificar si también hay diferencias significativas entre los tipos de espectadores de episodios de violencia entre pares, respecto a su capacidad de disfrute y su percepción de apoyo comunitario. 
Ahora bien, la capacidad de disfrute, o gaudibilidad, es una categoría disposicional que aún está en exploración, teniendo como antecedente el concepto de "susceptibilidad al reforzamiento”, de Gray (1987, 1993). Según esto, las personas con alta susceptibilidad suelen buscar reforzadores con mayor asiduidad que otros sujetos, mas la gaudibilidad resulta ser un constructo de mayor alcance, puesto que se asocia a la emoción degustadora que acompaña al refuerzo. El disfrute implica la posibilidad de "saborear el placer", así que la gaudibilidad es sinónimo de capacidad de (o para el) disfrute y se postula que tiene mucha relevancia terapéutica (Montgomery, 2009). En suma, de acuerdo con Csikszentmihalyi (Cuadra y Florenzano, 2003) en términos operativos la sensación de disfrute aparecería cuando las personas perciben situaciones como desafíos y a la vez se autoperciben como capacitadas para superarlos, por lo que la gaudibilidad es la potencialidad para experimentar sensaciones gratificantes; es decir, el refinamiento en la ejecución de actividades (tanto las que se hacen para lograr un objetivo como las que se realizan por el placer de hacerlas). Esta capacidad es modulada por las creencias, habilidades, valoraciones y calidad de vida de las personas.

Por otro lado, la interacción social es un potencial fuente de apoyo para las personas. Existen tres niveles de vinculación social: la comunidad, las redes sociales y las relaciones íntimas y de confianza entre las personas. Gracia y Herrero (2004) acuñan el concepto de apoyo comunitario percibido que incluye: la identidad en función a la comunidad de pertenencia, la comunidad como fuente de seguridad y predictibilidad, y como referente para la construcción del autoconcepto y la autoestima; encontrando que en niveles mayores está relacionado con indicadores de ajuste psicológico.

Finalmente, se considera la violencia entre pares (bullying) a la actividad de tipo agresivo que algunos escolares - apoyados por la inhibición o tolerancia, e inclusive el aliento, del grupo- aplican repetida y deliberadamente sobre otros alumnos de forma sistemática, utilizando modalidades físicas, verbales y sociales. La dinámica de la violencia entre pares es la relación establecida entre los tres actores que participan en la situación de maltrato: agresor, víctima y espectador, caracterizándose por una asimetría en la cual los agresores intentan someter a la víctima y convertir al espectador en un cómplice activo o silencioso que tolera esta situación (Avilés, 2006).

El agresor suele ser un individuo con fortaleza física mayor, con falta de empatía afectiva, sin sentimientos de culpa, muestra un estilo atribucional externo, no es socialmente competente, trasgresor, con estilo agresivo activo al interrelacionarse intimidando directa o indirectamente. Por el contrario, la víctima es de menor fortaleza física, puede incluso presentar discapacidades, diferencias por pertenecer a minorías étnicas o sociales, con baja autoestima, timidez, inseguridad e inhabilidad social, careciendo a menudo de una red de apoyo, con características sumisas, pasivas, dependientes e inasertivas, con fracaso escolar, y en algunos casos pudiendo mostrar conductas provocadoras o desafiantes. Los espectadores usualmente identifican víctimas y agresores, mostrando incluso algunos de ellos una actitud complaciente ante la inequidad, mientras que otros son indiferentes, tolerantes ante estas situaciones injustas; sin embargo, existe también un grupo de estos que se muestran solidarios con la víctima. Al respecto, en la investigación anterior (Quintana, Montgomery y Malaver, 2009) se distinguieron tres tipos de espectadores de violencia entre 
pares: 1) prosocial, observador y cooperador con la víctima, 2) indiferente culpabilizado, observador pasivo-evitativo y amoral, y 3) consentidor con el agresor.

En consecuencia, el presente estudio busca, por medio de la indagación ya puntualizada a través de los conceptos operacionalizados mediante los instrumentos de evaluación respectivos, contrastar las hipótesis de que: 1) existen diferencias significativas entre los tipos de espectadores de episodios de violencia entre pares, respecto a la capacidad de disfrute y la percepción subjetiva de apoyo comunitario, 2) existe una covariación positiva significativa entre la capacidad de disfrute y el grado de integración en la comunidad; entre la capacidad de disfrute y el grado de participación en la comunidad; entre la capacidad de disfrute y la implicación en organizaciones comunitarias, y, por último, entre la capacidad de disfrute y la percepción de apoyo comunitario en general.

\section{MÉTODO}

\section{Diseño de investigación}

La primera hipótesis se contrastó mediante un diseño de tipo descriptivo-comparativo y transversal. Para contrastar la segunda hipótesis se utilizó un diseño descriptivocorrelacional.

\section{Población y muestra}

La muestra está conformada por 400 estudiantes adolescentes tanto de género masculino, como femenino, representativos de los tres tipos identificados de espectadores de la violencia entre pares. Seleccionados mediante la técnica de muestreo no probabilística: muestreo intencional. Las variables del estudio son la capacidad de disfrute (gaudibilidad), la percepción subjetiva de apoyo comunitario en la escuela y los tipos de espectador de violencia entre pares. Como variables controladas figuran el nivel socio-económico: medio bajo, edades: entre 14-18 años, grado de instrucción: secundaria, y lugar de residencia: los distritos de Cercado de Lima y San Juan de Lurigancho.

\section{INSTRUMENTOS}

La ficha demográfica. Cuestionario que permite registrar algunas características relevantes de los sujetos estudiados: lugar de residencia, edad, sexo, grado de instrucción, etc.

La escala de gaudibilidad (Padrós, 2002). Es una escala unidimensional de 23 ítemes que mide la capacidad de disfrute, los que se responden con una escala likert de cinco puntos (totalmente de acuerdo, de acuerdo, indiferente, en desacuerdo y totalmente en desacuerdo). Para obtener el puntaje directo se suman las respuestas dadas. Respecto a la validez encontramos que la estructura interna se realizó a partir del análisis factorial de componentes principales, en el ajuste de la solución con un factor se observa que la variancia explicada del modelo es alrededor de un $25 \%$. Además, el estudio muestra una buena validez concurrente con calidad de vida, sensación de felicidad, bienestar subjetivo, 
etc. y la confiabilidad indica una alta consistencia interna con un alfa de Cronbach que oscila en las diferentes muestras poblacionales estudiadas desde 0.8383 a 0.8589 . La adaptación de la misma al ambiente escolar se consigna en el apéndice A.

El cuestionario de apoyo comunitario percibido (Gracia y Herrero, 2004). Proporciona una medida de la integración y participación comunitaria así como un índice del apoyo percibido en organizaciones voluntarias de la comunidad (grupos sociales, clubes, asociaciones deportivas, comisiones de festejos, etc.). El cuestionario se compone de tres subescalas con categorías de respuesta que van de "Muy en desacuerdo" (1) a "Muy de acuerdo" (5): integración comunitaria, de 4 ítemes; participación comunitaria, de 5 ítemes, y organizaciones comunitarias, de 5 ítemes. La validez se encontró mediante el análisis factorial de componentes principales, el cual mostró que tres componentes con autovalores mayor que uno, correspondientes a las tres escalas, se rotaron oblicuamente. La confiabilidad indica un alfa de Cronbach para la escala global $(a=0.87)$ y cada una de las dimensiones $\left(\mathrm{a}^{3}\right.$ 0.76). La adaptación de la misma al ambiente escolar se muestra en el apéndice $B$.

La escala de tipo de espectador de violencia entre pares (TEVEP). Utilizada por los autores en un estudio anterior (Quintana, Montgomery y Malaver, 2009), es una escala de 30 ítemes originales, calificados en función a su frecuencia: desde siempre (4) a nunca (1), y otros 5 ítemes extraídos de otros instrumentos de acoso entre iguales, a efectos de contextualizar la presencia de estos episodios, la cual permite identificar tres tipos de adolescentes espectadores de violencia entre pares: prosocial, indiferente-culpabilizado y antisocial. La validez probada por criterio de expertos arroja índices de coeficiente de validez de Aiken de los ítemes que oscilaron predominantemente entre 0.80 y 1.00 . La confiabiliadad obtenida por el método de ítemes pares/impares proporcionó un $\mathrm{r}=.555$ estadísticamente significativo al .001. Al momento de redactar esta entrega se realiza un estudio piloto para validar este instrumento mediante un análisis de su estructura factorial y probar su confiabilidad mediante el coeficiente alfa de Cronbach para la escala global y para cada uno de sus ítemes y dimensiones. Resultados se darán a conocer en un próximo artículo.

\section{RESULTADOS}

Respecto a la hipótesis acerca de la existencia de diferencias significativas entre los tipos de espectadores de episodios de violencia entre pares, en relación a la capacidad de disfrute y la percepción subjetiva de apoyo comunitario, la respuesta es que se observan diferencias estadísticamente significativas entre el tipo de espectador prosocial y los tipos de espectador amoral e indiferente-culpabilizado en cuanto a la percepción subjetiva de apoyo comunitario, no encontrándose diferencias significativas entre estos últimos (Figura 1, Tabla 1) . 


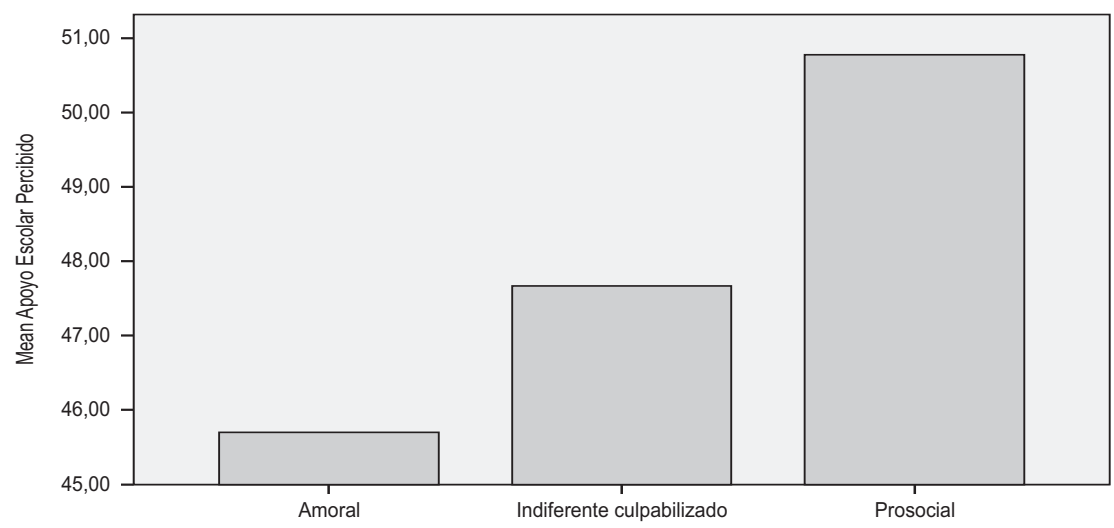

Figura N. ${ }^{\circ}$ 1. Apoyo escolar percibido por el tipo de espectador de violencia entre pares.

Tabla N. ${ }^{\circ}$ 1. Apoyo social percibido y tipos de espectadores de violencia entre pares.

Dependent variable: Apoyo escolar percibido. Scheffe

\begin{tabular}{|c|c|c|c|c|c|c|}
\hline \multirow{2}{*}{$\begin{array}{l}\text { (I) Tipo de espectador } \\
\text { de violencia entre pares }\end{array}$} & \multirow{2}{*}{$\begin{array}{l}\text { (J) Tipo de espectador } \\
\text { de violencia entre pares }\end{array}$} & \multirow{2}{*}{$\begin{array}{c}\text { Mean } \\
\text { difference (I-J) }\end{array}$} & \multirow{2}{*}{$\begin{array}{l}\text { Std. } \\
\text { error }\end{array}$} & \multirow[b]{2}{*}{ Sig. } & \multicolumn{2}{|c|}{$95 \%$ confidence interval } \\
\hline & & & & & Lower bound & Lipper bound \\
\hline \multirow{2}{*}{ Amoral } & Indiferente culpabilizado & $-1,96517$ & 1,11432 &, 213 & $-4,7101$ & ,7797 \\
\hline & Prosocial & $-5,07270 *$ & 1,26929 &, 000 & $-8,1993$ & $-1,9464$ \\
\hline \multirow{2}{*}{ Indiferente culpabilizado } & Amoral & 1,96517 & 1,11432 &, 213 &,- 7797 & 4,7101 \\
\hline & Prosocial & $-3,10753 *$ & 1,14200 &, 026 & $-5,9206$ &,- 2944 \\
\hline \multirow{2}{*}{ Prosocial } & Amoral & $5,07270^{*}$ & 1,26929 &, 000 & 1,9461 & 8,1993 \\
\hline & Indiferente culpabilizado & $3,10753 *$ & 1,14200 & ,026 &, 2944 & 5,9206 \\
\hline
\end{tabular}

*The mean difference is significant at the .05 level

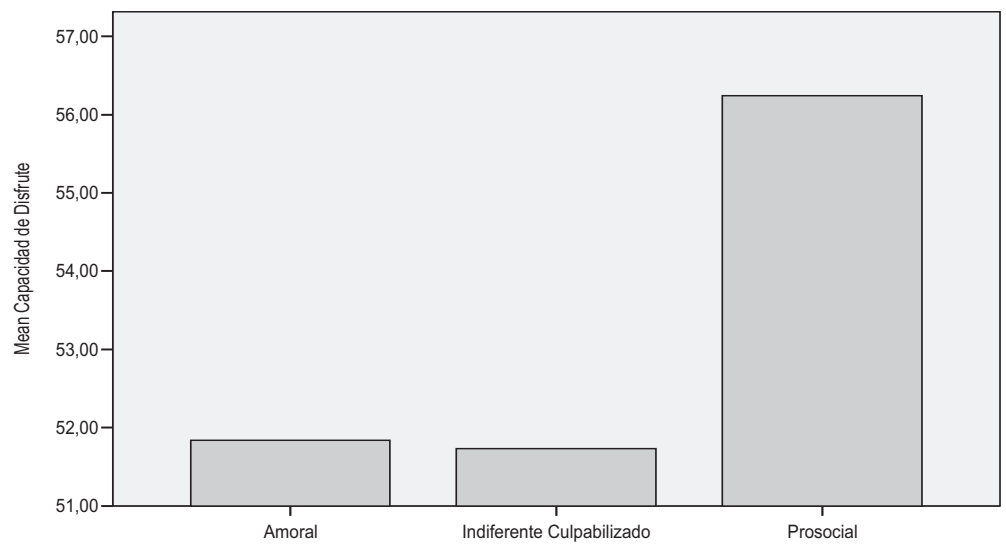

Figura N. ${ }^{\circ}$ 2. Capacidad de disfrute por el tipo de espectador de violencia entre pares. 
En la Figura 2 se observan resultados que no coinciden con el análisis de varianza correspondiente (Tabla 2), en el cual no se observan diferencias significativas respecto a la capacidad de disfrute entre los tipos de espectadores de violencia entre pares.

Tabla N. ${ }^{0}$ 2. Capacidad de disfrute y tipos de espectadores de violencia entre pares.

\begin{tabular}{|c|c|c|c|c|c|c|}
\hline \multirow{2}{*}{$\begin{array}{l}\text { (I) Tipo de espectador } \\
\text { de violencia entre pares }\end{array}$} & \multirow{2}{*}{$\begin{array}{l}\text { (J) Tipo de espectador } \\
\text { de violencia entre pares }\end{array}$} & \multirow{2}{*}{$\begin{array}{c}\text { Mean } \\
\text { difference (I-J) }\end{array}$} & \multirow{2}{*}{$\begin{array}{l}\text { Std. } \\
\text { error }\end{array}$} & \multirow[b]{2}{*}{ Sig. } & \multicolumn{2}{|c|}{$\mathbf{9 5 \%}$ confidence interval } \\
\hline & & & & & Lower bound & Lipper bound \\
\hline \multirow{2}{*}{ Amoral } & Indiferente culpabilizado & $-1,0609$ & 2,20913 & ,999 & $-5,3356$ & 5,5478 \\
\hline & Prosocial & $-4,40611$ & 2,51635 & ,218 & $-10,6046$ & 1,7924 \\
\hline \multirow{2}{*}{ Indiferente culpabilizado } & Amoral & $-1,0609$ & 2,20913 & ,999 & $-5,5478$ & 5,3356 \\
\hline & Prosocial & $-4,51221$ & 2,26399 &, 140 & $-10,0891$ & 1,0647 \\
\hline \multirow{2}{*}{ Prosocial } & Amoral & 4,40611 & 2,51635 &, 218 & $-1,7924$ & 10,6046 \\
\hline & Indiferente culpabilizado & 4,51221 & 2,26399 &, 140 & $-1,0647$ & 10,0891 \\
\hline
\end{tabular}

Sin embargo, la Tabla 3 expresa diferencias estadísticamente significativas (al 0.05) de la capacidad de disfrute, si comparamos aisladamente al tipo de espectador de violencia entre pares prosocial con el tipo indiferente-culpabilizado.

Tabla N. ${ }^{\circ}$ 3. Diferencia de medias de la capacidad de disfrute entre el tipo de espectador de violencia entre pares prosocial y el tipo indiferente culpabilizado.

\begin{tabular}{|c|c|c|c|c|c|c|c|c|c|c|}
\hline & & \multicolumn{4}{|c|}{$\begin{array}{l}\text { Levene's test } \\
\text { for equality } \\
\text { of variances }\end{array}$} & \multicolumn{3}{|c|}{ t-test for equality of means } & \multicolumn{2}{|c|}{$\begin{array}{l}\text { 95\% confidence } \\
\text { interval of the } \\
\text { difference }\end{array}$} \\
\hline & & $\mathbf{F}$ & Sig. & $\mathbf{t}$ & df & $\underset{\text { (2-tailed) }}{\text { Sig }}$ & $\begin{array}{c}\text { Mean } \\
\text { difference }\end{array}$ & $\begin{array}{l}\text { Std. error } \\
\text { difference }\end{array}$ & Lewer & Upper \\
\hline \multirow[t]{2}{*}{$\begin{array}{l}\text { Capacidad } \\
\text { de disfrute }\end{array}$} & $\begin{array}{l}\text { Equal variances } \\
\text { assumed }\end{array}$ &, 057 &, 811 & $-2,020$ & 171 &, 045 & $-4,51221$ & 2,23354 & $-8,92106$ &,- 10335 \\
\hline & $\begin{array}{l}\text { Equal variances } \\
\text { not assumed }\end{array}$ & & & $-2,056$ & 132,980 &, 042 & -451221 & 2,19479 & $-8,85342$ &,- 17099 \\
\hline
\end{tabular}

En cuanto a las hipótesis de que existirían correlaciones positivas significativas entre la capacidad de disfrute y la percepción subjetiva de apoyo comunitario, y sus distintos componentes, a saber: el grado de integración en la comunidad, el grado de participación en la comunidad y la implicación en organizaciones comunitarias, la Tabla 4 muestra que los resultados de los coeficientes de correlación de Pearson significativos al $0.01 \mathrm{y}$ 0.05 encuentran correlaciones positivas significativas con la integración a la comunidad escolar (0.148), con la participación en la comunidad escolar (0.198), con el apoyo de las organizaciones de la comunidad escolar $(0.173)$ y con la percepción subjetiva de apoyo comunitario en general (0.236). 
Tabla N. ${ }^{\circ}$ 4. Correlación entre la capacidad de disfrute, el grado de integración a la comunidad escolar, la participación en la comunidad escolar y el apoyo de las organizaciones de la comunidad escolar.

\begin{tabular}{|c|c|c|c|c|c|c|}
\hline & & $\begin{array}{l}\text { Capacidad } \\
\text { de disfrute }\end{array}$ & $\begin{array}{l}\text { Apoyo escolar } \\
\text { percibido }\end{array}$ & $\begin{array}{c}\text { Apoyo de la } \\
\text { organización } \\
\text { escolar }\end{array}$ & $\begin{array}{l}\text { Particpación } \\
\text { escolar }\end{array}$ & $\begin{array}{l}\text { Integración } \\
\text { escolar }\end{array}$ \\
\hline \multirow{3}{*}{ Capacidad de disfrute } & Pearson correlation & 1 &, $236 * *$ &, $173 * *$ &, $198 * *$ &, $148 * *$ \\
\hline & Sig. (2-tailed) & &, 000 &, 003 &, 001 &, 012 \\
\hline & $\mathrm{N}$ & 388 & 388 & 388 & 388 & 388 \\
\hline \multirow{3}{*}{ Apoyo escolar percibido } & Pearson correlation &, $236 * *$ & 1 &, $171^{* *}$ &, $793 * *$ &, $636^{* *}$ \\
\hline & Sig. (2-tailed) &, 000 & &, 000 &, 000 &, 000 \\
\hline & $\mathrm{N}$ & 388 & 388 & 388 & 388 & 388 \\
\hline \multirow{3}{*}{$\begin{array}{l}\text { Apoyo de la organización } \\
\text { escolar }\end{array}$} & Pearson correlation &, $173 * *$ & $-771 * *$ & 1 &, $406 * *$ &, $248 * *$ \\
\hline & Sig. (2-tailed) &, 003 &, 000 & &, 000 &, 000 \\
\hline & $\mathrm{N}$ & 388 & 388 & 388 & 388 & 388 \\
\hline \multirow{3}{*}{ Participación escolar } & Pearson correlation &, $198 * *$ &, $793 * *$ &, $406 * *$ & 1 &, $279 * *$ \\
\hline & Sig. (2-tailed) &, 001 &, 000 &, 000 & &, 000 \\
\hline & $\mathrm{N}$ & 388 & 388 & 388 & 388 & 388 \\
\hline \multirow{3}{*}{ Integración escolar } & Pearson correlation &, $148^{*}$ &, $636^{* *}$ &, $248 * *$ &, $279 * *$ & 1 \\
\hline & Sig. (2-tailed) &, 012 &, 000 &, 000 &, 000 & \\
\hline & $\mathrm{N}$ & 388 & 388 & 388 & 388 & 388 \\
\hline
\end{tabular}

** Correlation is siginificant at the 0.01 level (2-tailed).

* Correlation is siginificant at the 0.05 level (2-tailed).

\section{DISCUSIÓN}

A través de las mediciones correspondientes se verifican algunas relaciones entre los tipos de espectadores de episodios de violencia entre pares, en cuanto a la capacidad de disfrute y la percepción subjetiva de apoyo comunitario. En particular, como era de esperar, aparecen diferencias estadísticamente significativas entre el tipo de espectador prosocial y los tipos de espectador amoral e indiferente-culpabilizado respecto a la percepción subjetiva de apoyo comunitario. Sin duda tiene que ver con esto el hecho de que en la actitud prosocial juega un papel importante percibir que en circunstancias conflictivas se tiene en quien confiar y a quien solicitar apoyo, lo cual, como señala Barraza (2008), puede reducir la magnitud estresora de un evento aversivo.

También se puede apreciar que hay diferencias significativas entre los tipos de espectador de violencia entre pares "prosocial" e "indiferente culpabilizado", en cuanto a la capacidad de disfrute. Esto hace pensar que el espectador indiferente culpabilizado carece de humor y sentimiento de bienestar, lo que está en una dimensión de afecto negativo opuesta a la gaudibilidad. Asimismo, dicha dimensión de afecto negativo, de acuerdo con estudios motivacionales clásicos (ver Palmero, 2002), se correlaciona con alto arousal o estado de activación típico, en este caso, de los estados de tensión. No es extraño, pues, que el espectador indiferente o culpabilizado rehuya "meterse en problemas ajenos" en una situación de observación de acoso. 
Por último, los resultados muestran que la gaudibilidad y la percepción de apoyo comunitario se relacionan en cualquiera de sus componentes, lo que enfatiza la pertinencia de un marco sistémico mediante el cual analizar los factores de acoso, los tipos de espectador de acoso y el ambiente institucional. Los datos señalan que a mayor integración, participación y apoyo recibido por la comunidad escolar (variables que podrían considerarse globalmente como "apoyo social"), hay mayor predictibilidad de bienestar subjetivo (Gracia y Herrero, 2006), y mayor posibilidad de cooperación en las aulas (Díaz-Aguado, 2006).

\section{CONCLUSIONES}

Desde la perspectiva mantenida en este estudio y en el anterior (Quintana, Montgomery y Malaver, 2009), sin duda el papel del espectador prosocial es gravitante debido a su presencia inmediata a la ocurrencia del problema del acoso, y debe ocupar un lugar preferente en la postulación de soluciones para este problema socioescolar.

En este sentido, desde ya resulta prioritario aumentar el apoyo social y las redes sociales en la escuela, además de fomentar el incremento de espectadores de tipo prosocial.

Ahora bien, en el entendido de que el estilo explicativo que cada cual argumenta sobre su responsabilidad en el desarrollo de los acontecimientos está ligado con su propia capacidad de manejo de conflictos interpersonales (San Juan y Magallares, 2007), resulta de rigor determinar qué estilos explicativos se encuentran en la base del sentimiento que expresa el espectador prosocial acerca de su capacidad de evitar los conflictos interpersonales o de intervenir de alguna manera para menguarlos, y qué estilos explicativos subyacen a la tolerancia o aliento que manifiestan otros tipos de espectadores hacia el acoso.

De otro lado, resulta interesante conocer, así mismo, si esos estilos explicativos guardan relación con las habilidades para la gestión de conflictos que tienen uno u otro grupo de escolares, en consonancia con la conceptualización de Staats (1997).

\section{NOTA DE RECONOCIMIENTO}

El responsable reconoce a los siguientes investigadores: Carlos Arenas I., Pedro García P., Elisa Yánac R. y Augusto Frisancho L., por compartir la autoría de esta investigación y, así mismo, a los alumnos Nayú García Z., Eduardo Moras R. y Daysi Aponte A., por su desinteresada y esmerada colaboración en este estudio.

\section{REFERENCIAS BIBLIOGRÁFICAS}

1. Avilés, J. (2006). Bullying: el maltrato entre iguales. Salamanca: Amaru.

2. Barraza, A. (2008). El estudio de la red de apoyo social en alumnos de educación media superior. En Revista electrónica de psicologia cientifica. com. Recuperado de: http://www.psicologiacientifica.com/bv/ psicologia-332-1-el-estudio-de-la-redde-apoyo-social-en-alumnos-de-educacion.html.

3. Blanco, A. y Díaz, D. (2006). Orden social y salud mental: una aproximación desde el bienestar social. En Clínica y salud, 17, 1, 7-29. 
4. Cuadra, H. y Florenzano, R. (2003). El bienestar subjetivo: hacia una psicología positiva. En Revista de psicología de la Universidad de Chile, 12, 1, 83-96.

5. Díaz-Aguado, M. (2006). Del acoso escolar a la cooperación en las aulas. Madrid: Pearson.

6. Gracia, E. y Herrero, J. (2004). Determinants of social integration in the community: an exploratory analysis of personal, interpersonal, and situational variables. En Journal of community and applied social psychology, 14, 1-15.

7. Gracia, E. y Herrero, J. (2006). La comunidad como fuente de apoyo social: evaluación e implicaciones en los ámbitos individual y comunitario. En Revista latinoamericana de psicología, 38, 2, 327-342.

8. Gray, J. A. (1987/1993). La psicología del miedo y el estrés. Barcelona: Labor.

9. Montgomery, W. (2009). Mindfullnes y gaudibilidad: categorías en terapia de conducta para tratar la evitación experiencial. En Revista de psicología de la Universidad Garcilazo de la Vega, 1, 1, 57-61.

10. Padrós, F. (2002). Disfrute y bienestar subjetivo. Un análisis psicométrico de la gaudibilidad, (tesis de doctorado en psicología). Barcelona: Universidad de Barcelona.

11. Padrós, F. y Fernández, J. (2002). Escala de gaudibilidad de Padrós. Una propuesta para medir la disposición a experimentar bienestar. En Boletín de psicología, 71, $7-28$.

12. Palmero, F. (2002). Psicología de la motivación y la emoción. Madrid: McGrawHill.

13. Quintana, A.; Montgomery, W. y Malaver, C. (2009). Modos de afrontamiento y conducta resiliente en adolescentes expectadores de violencia entre pares. En Revista de psicología de la Universidad Nacional Mayor de San Marcos, 12, 1, 153-172.

14. Salotti, P. (2006). Estudio sobre estrategias de afrontamiento y bienestar psicológico en una muestra de adolescentes de Buenos Aires, (tesis de licenciatura en psicología). Buenos Aires: Universidad de Belgrano.

15. Seligman, M. y Csikszentmihalyi, M. (2000). Positive psychology: an introduction. En American Psychologist, 55, 1, 5-14.

16. San Juan, P. y Magallares, A. (2007). Estilos explicativos y estrategias de afrontamiento. En Clínica y salud, 18, 1, 83-98.

17. Staats, A. W. (1997). Conducta y personalidad: conductismo psicológico. Bilbao: Descleé de Brouwer.

18. Vázquez, C.; Hervás, G. y Ho, S. (2006). Intervenciones clínicas basadas en la psicología positiva: fundamentos y aplicaciones. En Psicología conductual, 14, 3, 401-432. 